
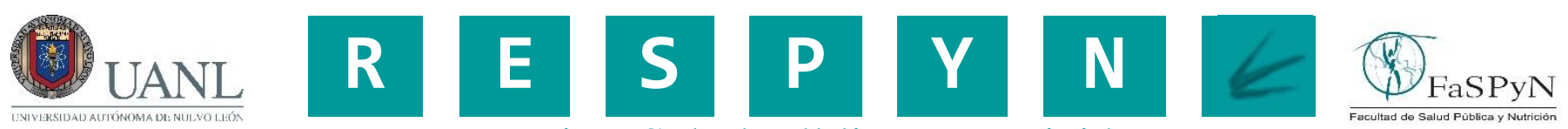

Revista Salud Pública y Nutrición

\title{
LA OBESIDAD DESDE LA PERSPECTIVA DE LA SELECCIÓN DE ALIMENTOS.
}

\author{
OBESITY FROM THE PERSPECTIVE OF FOOD SELECTION. \\ Arriaga-Ramírez José Cristóbal Pedro*, Cruz-Morales Sara E.* \\ * Universidad Nacional Autónoma de México, México.
}

Citation: Arriaga-Ramírez J.C.P., Cruz-Morales S.E. (2019) La obesidad desde la perspectiva de la selección de alimentos. Revista de Salud Pública y Nutrición, 18 (1), 25-32.

Editor: Esteban G. Ramos Peña, Dr. CS., Universidad Autónoma de Nuevo León, Facultad de Salud Pública y Nutrición, Monterrey Nuevo León, México. Copyright: (C2019 Arriaga-Ramírez J.C.P. et al. This is an open-access article distributed under the terms of Creative Commons Attribution License [CC BY 4.0], which permits unrestricted use, distribution, and reproduction in any medium, provided the original author and source are credited.

Competing interests: The authors have declared that no competing interests exist.

DOI: $\underline{\text { https://doi.org/10.29105/respyn18.1-4 }}$

Recibido: 08 de enero 2019;

Aceptado: 20 de marzo 2019

Email: jcpedro@unam.mx 


\title{
LA OBESIDAD DESDE LA PERSPECTIVA DE LA SELECCIÓN DE ALIMENTOS
}

\author{
Arriaga-Ramírez José Cristóbal Pedro*, Cruz-Morales Sara E.*
}

* Universidad Nacional Autónoma de México, México.

\section{RESUMEN}

Introducción: La obesidad es un problema de salud a nivel mundial, así como en México. La iniciación de la alimentación se ha estudiado desde la perspectiva de la homeostasis. El aprendizaje es un mecanismo de adaptación que permite la regulación de la alimentación en ausencia de señales de error, como lo implican las estrategias basadas en cambios de las variables reguladas homeostáticamente y provee un mecanismo flexible y dinámico especialmente en ambientes predecibles. Objetivo: La tesis de este trabajo es que, mediante la selección de alimentos, mediada socialmente como influencia directa por medio de la enseñanza, para elegir, comprar y preparar los alimentos de una lista en donde se indique el contenido de carbohidratos, se puede ayudar a resolver el problema de la obesidad causada por el consumo excesivo de éstos. Conclusiones: La selección de alimentos con base en la influencia social puede proveer estrategias para controlar la obesidad mediante la limitación en el consumo de carbohidratos. Se ha encontrado que las dietas bajas en carbohidratos producen una disminución de peso mayor que la limitación del contenido de grasa en la dieta. En las dietas bajas en carbohidratos, una variedad efectiva en la reducción del peso es la dieta cetogénica.

Palabras Clave: Obesidad, alimentación, aprendizaje social.

\section{ABSTRACT}

Introduction: Obesity is a health problem worldwide and in México. Feeding initiation has been studied from a homeostasis view. Learning is an adaptive mechanism that allows feeding regulation in absence of error signals as implied by strategies based on disturbances on homeostatic guided variables and gives a flexible and dynamic mechanism specially in predictive environments. Objective: The thesis of this work is that, by means of food selection, socially guided as a direct influence by means of teaching, to choose, buy, and to prepare foods from a list where the amount of carbohydrates is shown, may help to solve the obesity problem caused by excessive consumption of them. Conclusions: Food selection with base on social influences and may provide strategies to reduce obesity by limiting the amount of carbohydrates consumed. It has been found that low carbohydrate diets reduce weight more than the reduction of fat in diet. In low carbohydrate diets, one that has been found effective is the ketogenic diet.

Key words: Obesity, feeding, social learning. 


\section{Introducción}

La tesis de este trabajo es que, mediante la selección de alimentos, mediada socialmente como influencia directa por medio de la enseñanza, para elegir, comprar y preparar los alimentos de una lista en donde se indique el contenido de carbohidratos, se puede ayudar a resolver el problema de la obesidad causada por el consumo excesivo de éstos.

La obesidad es un problema de salud a nivel mundial, en México y en países en desarrollo (Imes \& Burke, 2014; Swinburn et al., 2011; WHO, 2018). En México el $42.6 \%$ de los hombres mayores de 20 años, presentan sobrepeso y $26.8 \%$ obesidad. El $35.5 \%$ de las mujeres mayores de 20 años, presentan sobrepeso y $35.2 \%$ obesidad. En el país, aproximadamente 7 de cada 10 adultos tienen exceso de peso (Gutiérrez, et al., 2013). Desde la Psicología Experimental un área que tiene estrategias para ayudar a resolver este problema es el de la Selección de Alimentos. En este trabajo se presentan algunos determinantes del inicio de la alimentación, factores que guían la selección de alimentos, la participación del aprendizaje individual y mediado socialmente en la selección de alimentos, datos acerca de la utilidad de las diferentes estrategias como ejercicio, dietas y algunas sugerencias con base en la participación del aprendizaje mediado socialmente para resolver el problema de la obesidad.

Determinantes psicológicos del inicio de la alimentación. El comer es una de las actividades humanas más básicas (Capaldi, 1996). En la Psicología un área que estudia por qué comemos lo que comemos es la de la Selección de Alimentos, el estudio de la selección de alimentos con influencia social resultó importante porque como lo indicó el trabajo de Galef (1991), ésta no ocurre en el medio natural de manera óptima como lo sugieren algunas aproximaciones teóricas. La selección de los alimentos está determinada por el ambiente, las consecuencias orgánicas de los alimentos, la influencia social a que está sujeto el que se alimenta que puede guiar el consumo de alimentos seguros y evitar alimentos dañinos. La iniciación de la alimentación, en la Psicología, se ha descrito proponiendo varias estrategias. Una de ellas es la homeostasis, que consiste en el mantenimiento del equilibrio en diversos parámetros fisiológicos (Bernard 1878, citado en Ramsay, Seeley, Bolles \& Woods, 1996). El mecanismo que opera en este mantenimiento del equilibrio es uno de retroalimentación negativa (McFarland, 1971), en ella un nivel establecido de los parámetros se mantiene estable, cuando el sistema detecta un cambio en ese parámetro se procede a consumir alimento para regresar al nivel preestablecido. Sin embargo, la estrategia de la retroalimentación negativa tiene aspectos criticables. Uno de ellos es que se debe producir una señal de error para que se genere una respuesta de corrección del parámetro (Ramsay et al., 1996). Otro mecanismo propuesto es el de la regulación anticipatoria y se supone que éste evita el problema de los mecanismos de retroalimentación negativa (Houk, 1988). Esta estrategia implica que los organismos tengan un decodificador de los nutrientes de cada alimento que requieren (Ramsay et al., 1996). Sin embargo, esta estrategia sugiere que se debe dar una "corrección" antes de que se produzca un desequilibrio (Houk, 1988). En ambas estrategias se requiere de un detector de la condición de error cuando ocurre o antes de que ocurra. Como es evidente, no es claro en qué consisten o como pueden indicar al que se alimenta estos detectores de error en los parámetros que determinan el inicio de la alimentación.

Otro mecanismo necesario para explicar el inicio de la alimentación y la selección de los alimentos. En la selección de alimentos se involucra el aprendizaje (Birch \& Fisher, 1996). El aprendizaje es un mecanismo de adaptación que permite una regulación de la alimentación en ausencia de señales de error como lo implican las dos estrategias mencionadas antes. En el aprendizaje de preferencias alimenticias el sabor del alimento se asocia con sus efectos. Si un alimento tiene efectos positivos se consumirá más en el futuro; en cambio sí tiene efectos negativos se generará una aversión que hará que se evite consumirlo (Rozin, 1996). El aprendizaje provee un mecanismo flexible $y$ dinámico mediante el cual se evita atribuir la iniciación de la alimentación a las perturbaciones en las variables reguladas homeostáticamente, especialmente en ambientes predecibles, en los que se puede anticipar las perturbaciones a la homeostasis (Ramsay et al., 1996). Específicamente, el consumo de los alimentos dependerá de las condiciones ambientales y sociales que rodean al consumo de estos que puede cambiar, por medio del aprendizaje, si las condiciones cambian. El aprendizaje permite elegir alimentos seguros para 
mantener la salud, a través de la formación de relaciones entre el consumo y sus efectos. Es necesario depender del aprendizaje individual y social porque, como algunas aproximaciones teóricas lo sugieren, la selección de alimentos no ocurre de manera automática en el medio natural ni en condiciones de laboratorio como lo muestran los estudios en los que se ha analizado la selección de alimentos con situaciones de tipo "cafetería" en las que se presenta una variedad de alimentos con diferentes nutrientes (Galef, 1991).

\footnotetext{
Algunos mecanismos de aprendizaje que pueden explicar la conducta de alimentarse. El aprendizaje puede tener la forma de condicionamiento clásico y se pueden generar tanto aversión (Schafe \& Bernstein, 1996) como preferencia por diversos alimentos (Capaldi, 1996). La preferencia de alimentos puede generarse por diversos procedimientos de condicionamiento simple o individual, por ejemplo, el efecto medicinal, asociaciones sabor-sabor, sabor-nutrientes, el efecto postre, etc. (Capaldi, 1996). El efecto medicinal genera una preferencia por alimentos consumidos durante la recuperación de una enfermedad. Las asociaciones sabor-sabor se producen cuando un sabor preferido, como los sabores dulces, se asocia con un alimento novedoso y como resultado se genera una preferencia por el alimento novedoso. Las asociaciones sabor-nutrientes se producen cuando un sabor novedoso se asocia con los efectos de las calorías de un alimento preferido, como por ejemplo la sacarosa. El efecto postre ocurre cuando un alimento novedoso es seguido de un alimento preferido como la sacarosa. La selección de alimentos tiene unas bases asentadas en el condicionamiento simple y en el condicionamiento basado en la influencia social (Birch \& Fisher, 1996; Rozin, 1996). Esta influencia social puede ser directa, en este tipo se requiere la mediación de otro individuo de la misma especie. La influencia también puede ser de manera inadvertida, en este caso la presencia social directa de otro individuo es necesaria pero no está orientada a propósito para producir un efecto determinado. En el tipo de influencia de una agencia social activa el agente social, un individuo, participa en la tarea de aprendizaje como un maestro activo. Este tipo de influencia es la que se requiere para enseñar a diferenciar los alimentos abundantes en carbohidratos para superar el gusto "innato" por los
}

sabores dulces (Cowart, 1981; Rozin, 1996). El aprendizaje mediado socialmente puede influir en la selección de alimentos dependiendo de la valencia del contexto social en el que se presentan, si es positiva como en una reunión con familiares o amigos puede generar preferencia; si es negativa como cuando se fuerza a los niños a comer alimentos porque son "buenos para ellos", puede generar una disminución en el consumo o aversión (Birch \& Fisher, 1996). El aprendizaje con la participación de un maestro activo también puede ocurrir enseñando a seleccionar alimentos para consumir usando una lista de alimentos y formando una discriminación entre alimentos seguros y alimentos dañinos. Este tipo de aprendizaje es de tipo instrumental u operante mediado socialmente. A través de este procedimiento pueden producirse preferencias de alimentos con los nutrientes adecuados para mantener la salud y evitar por ejemplo el consumo excesivo de carbohidratos y la obesidad. Este tipo de aprendizaje está mediado por un agente social que participa como maestro en la tarea de aprendizaje (Birch \& Fisher, 1996; Rozin, 1996).

La selección de alimentos puede resolver el problema que se observa en la explicación tradicional de la obesidad como exceso de comida y falta de ejercicio. En el área de la salud a nivel mundial un aspecto relacionado estrechamente con la selección de alimentos y el aprendizaje que parece tener proporciones de epidemia es el incremento en obesidad (Imes \& Burke, 2014; Swinburn et al., 2011; WHO, 2018), de igual manera que ocurre en México (Gutiérrez, et al., 2013) y en otros países en desarrollo. La lógica tradicional explica el aumento de peso como una diferencia entre las calorías ingeridas mediante la alimentación y las calorías gastadas a través de la actividad física. Esta relación puede estudiarse desde la perspectiva psicológica. Solamente que se deben hacer algunas precisiones. La relación entre calorías ingeridas y gastadas se ha explicado desde la perspectiva de la física como una relación de las leyes de la termodinámica que sugiere que si se mantiene un equilibrio entre estas cantidades consumidas y gastadas se puede entender la producción de la obesidad o la ausencia de ésta. Otra aclaración pertinente es que el organismo no es simplemente un ente físico como lo implica la afirmación anterior. Las calorías del alimento provienen de los macronutrientes: carbohidratos, grasas y proteínas. Los carbohidratos son 
monosacáridos como la glucosa o fructosa, o polisacáridos como la maltodextrina o las féculas. Las grasas son triglicéridos que son ácidos grasos, esteres del glicerol; la naturaleza de las cadenas alifáticas determina qué tipo de grasas son, por ejemplo, saturadas. Las proteínas, son cadenas de aminoácidos, 20 de los cuales ocurren naturalmente (Rowland, Li, \& Morien, 1996). Aunque en términos físicos el contenido energético de estos macronutrientes son calorías y una caloría es una caloría y la obesidad parece obedecer a un desequilibrio entre el consumo y el gasto de ellas. En el organismo los elementos de estos alimentos tienen efectos no solamente físicos sino también bioquímicos. Desde una perspectiva bioquímica los efectos de las "calorías", de los diferentes tipos de alimentos son diferentes. Cada uno de estos alimentos se metaboliza de manera diferente (Feinman \& Fine, 2003). Por esta razón no aplica la lógica de que una caloría es una caloría independientemente del macronutriente que provenga y tampoco que la obesidad se explica cuando se rompe el equilibrio entre entradas y salidas de éstas (Taubes, 2007). Además, esta razón no siempre se cumple, por ejemplo, la disminución de calorías consumidas no se acompaña con una disminución en el peso de acuerdo con un estudio realizado en Inglaterra (Griffit, Lluberas, \& Luhrmann, 2013). En algunos estudios no se ha encontrado que la actividad física prevenga el aumento de peso en los participantes (Church et al., 2009; Dwyer-Lindgren et al., 2013; I-Min, Djoussé, Sesso, Lu, \& Buring, 2010). Sin embargo, en otros sí se ha encontrado una reducción de peso cuando se ejercitaron de $370 \mathrm{~min} / \mathrm{semana}$ a $295 \mathrm{~min} / \mathrm{semana}$, y perdieron $1.4 \mathrm{y} 1.8 \mathrm{~kg}$ en promedio, mujeres y hombres respectivamente, en comparación al grupo control que aumentó $0.7 \mathrm{~kg}$ en mujeres y disminuyó $0.1 \mathrm{~kg}$ en hombres, durante un periodo de 12 meses en un estudio aleatorizado (McTiernan et al., 2007). Donelly et al. (2013) evaluaron en un estudio aleatorizado el efecto del ejercicio sobre la reducción de peso en un diseño de tres grupos. Un grupo control sin ejercicio, un grupo con ejercicio de reducción de $400 \mathrm{kcal} / \mathrm{sesión}$ y otro de $600 \mathrm{kcal} / \mathrm{sesión}$. Los dos grupos con ejercicio redujeron el peso durante los 10 meses que duró el estudio. Los resultados mostraron un aumento de $0.5 \%$ en el grupo control, y una disminución $4.3 \%$ y $5.7 \%$ en los grupos experimentales respectivamente.
Otro aspecto importante para considerar es que el organismo tiene un indicador del cambio en el peso y del consumo de energía. Cuando se aumenta o disminuye el peso, se establecen cambios compensatorios en el gasto de energía en el cuerpo, y estos se presentan al haber cambios de disminución de entre el $10 \%$ y el $20 \%$ del peso inicial (Leibel, Rosenbaum, \& Hirsch, 1995). Estos cambios compensatorios persisten seis años después de la pérdida de peso (Fothergill et al., 2016).

Estudios en los que se indica que el principal determínate de la obesidad es el consumo excesivo de carbohidratos. Diferentes autores han mencionado que uno de los generadores de la obesidad es el exceso de azúcar agregada a los alimentos procesados (Fung, 2016; Lustig, 2012, 2013, 2017; Taubes, 2007; Wilson \& Lowery, 2017). Algunos de estos autores indican que los determinantes del consumo de alimentos y la obesidad son las hormonas (Fung, 2016; Lustig, 2012, 2013, 2017). En particular los niveles de insulina producidos por el consumo de azúcar y carbohidratos en general. Sin embargo, podría cuestionarse si la selección de alimentos puede, al limitar el consumo excesivo azúcar en particular y de carbohidratos, limitar de alguna manera la producción de ciertas hormonas, específicamente la insulina y ayudar a controlar la obesidad. Los alimentos procesados tienen azúcar agregada y contribuyen a la obesidad. De acuerdo con Lustig (2012, 2017), la industria alimenticia ha modificado los alimentos para mantener su duración en los anaqueles eliminando la fibra natural y la grasa para anunciarlos como "reducidos en grasa". Esta manipulación causa deterioro del sabor en los alimentos y para compensar esto se agregan grandes cantidades de azúcar. Otro problema es que el azúcar tiene una gran cantidad de nombres equivalentes, cincuenta y seis, y no es fácil de identificar en las etiquetas de alimentos procesados (Lustig, 2013).

Evidencia de que la dieta baja en carbohidratos produce una disminución de peso mayor que la dieta baja en grasa. Algunos estudios controlados y aleatorizados han mostrado que la limitación en la cantidad de carbohidratos produce una disminución de peso mayor que la limitación del contenido de grasa en la dieta (Brehm, Seeley, Daniels, \& D'Alessio, 2003; Samaha et al., 2003; Shai et al., 2009; Sumithran et al., 2013; Yancy et al., 2004). 
Brehm et al. (2003) en un estudio aleatorizado de seis meses de duración encontraron que la dieta baja en grasa produjo una disminución de $2.0 \mathrm{~kg}$ y una dieta baja en carbohidratos produjo una disminución de $4.8 \mathrm{~kg}$. Los autores señalaron que la dieta baja en carbohidratos resultó mejor que la dieta baja en grasa para reducir el peso a corto plazo, seis meses, sin elevar el riesgo cardiovascular en mujeres sanas. Samaha et al. (2003) llevaron a cabo un estudio en el que compararon dos dietas, una baja en grasa y otra baja en carbohidratos. Al cabo de seis meses el grupo que perdió más peso fue el de la dieta baja en carbohidratos, $1.9 \mathrm{~kg}$ en el grupo con dieta baja en grasa y $5.8 \mathrm{~kg}$ en el grupo con dieta baja en carbohidratos. Shai et al. (2009) realizaron un estudio con duración de dos años. Compararon tres dietas: baja en grasa, Mediterránea y baja en carbohidratos. La pérdida de peso fue de $2.9 \mathrm{~kg}$ para la dieta baja en grasa, $4.4 \mathrm{~kg}$ para la dieta Mediterránea y $4.7 \mathrm{~kg}$ para la dieta baja en carbohidratos. Los autores concluyeron que la dieta Mediterránea y la dieta baja en carbohidratos son una alternativa efectiva para la reducción de peso corporal en comparación con la dieta baja en grasa. Sumithran et al. (2013) evaluaron la reducción de peso mediante una dieta baja en carbohidratos. Encontraron que los participantes redujeron el 13\% del peso inicial y que algunas hormonas y nutrientes que afectan el apetito se alteraron durante la dieta en comparación con la fase en la que se eliminó ésta. Yancy et al. (2004) compararon una dieta baja en grasa contra una dieta baja en carbohidratos en un estudio aleatorizado con 24 semanas de duración. Los sujetos del grupo de dieta baja en carbohidratos redujeron el $12.8 \%$ del peso contra los sujetos del grupo de la dieta baja en grasa sólo el $6.7 \%$.

De manera opuesta, otros estudios han encontrado una disminución de peso semejante para las dietas en las que se comparan los tres tipos de macronutrientes (Sacks et al., 2009).

Efectos positivos de la dieta baja en carbohidratos. En las dietas bajas en carbohidratos, una variedad de éstas que se ha encontrado efectiva en reducción de peso es la dieta cetogénica (Paoli, 2014; Wilson \& Lowery, 2017). La dieta cetogénica se caracteriza por una reducción en el contenido de carbohidratos a $50 \mathrm{~g}$ o menos por día y por un incremento de los otros macronutrientes grasas y proteínas (Paoli, Rubini, Volek, \& Grimaldi, 2013).
La dieta cetogénica tiene diversos efectos, uno de los principales para el tratamiento de la obesidad es la reducción del apetito (Gibson et al., 2015). Otros efectos terapéuticos de la dieta cetogénica son: la terapia en epilepsia una disminución de crisis (Lutas \& Yellen, 2013), en la diabetes reducción de la glucosa en la sangre (Sainbury et al., 2018), el síndrome de ovarios poliquísticos (Marvopoulos, Yancy, Hepburn, \& Westman, 2005), acné, enfermedades neurológicas como el Parkinson y Alzheimer (Kirkorian, Shidler, Dangelo, Couch, Benoit, \& Clegg, 2012; Taylor, Sullivan, Mahnken, Burns, \& Swerdlow, 2018) cáncer (Fine et al, 2012), y la reducción de riesgo en enfermedades respiratorias (Rubini et al., 2015) y riesgos cardiovasculares (Paoli et al., 2013; Volek et al., 2009). Otro aspecto que se ha observado en las dietas bajas en carbohidratos es una mejora en el perfil de lípidos de los participantes (Brehm et al., 2003; Paoli et al., 2013; Shai et al., 2008; Volek et al., 2009).

\section{Conclusiones}

Con base en la información mencionada en los apartados anteriores, la selección de alimentos puede utilizarse para resolver el problema de la obesidad cuando sea causada por el consumo excesivo de carbohidratos. En el tipo de influencia de una agencia social activa, el agente social participaría en la tarea de aprendizaje como un maestro activo (Rozin, 1996), como se mencionó antes. La estrategia por seguir, por parte del maestro activo, sería elaborar una lista de alimentos en la que se identifiquen los 56 nombres que tiene el azúcar en sus diferentes formas (Lustig, 2013) y los alimentos con gran contenido de carbohidratos, pues se ha demostrado que un cambio en la forma de alimentarse reduciéndolos, produce una reducción de peso (Fung, 2016; Lustig, 2012, 2013, 2017; Taubes, 2007; Wilson \& Lowery, 2017), así como enlistar los alimentos que contengan bajas cantidades de estos. Además del azúcar, el maestro activo puede identificar los alimentos con harina de trigo, féculas y otros elementos semejantes. Esta lista se usaría para enseñar a los sujetos con obesidad, que no tenga su origen en un padecimiento médico, a identificar el contenido de carbohidratos de los alimentos, a seleccionarlos, a guiar la compra de alimentos examinando la etiqueta y para la preparación de alimentos de acuerdo con recetas que pueden encontrarse en la literatura (por ejemplo, véase Fung, 2016; Lustig, 2012, 2013, 2017; Wilson \& Lowery, 2017). La meta por seguir sería reducirlos 
a un nivel que permita la pérdida de peso, por ejemplo, menos de $50 \mathrm{~g}$ al día y lograr un cambio duradero o permanente en la forma de alimentación y controlar la obesidad.

\section{Bibliografía}

Birch, L. \& Fisher, J. A. (1996). The role of experience in the development of children's eating behavior. In E. D. Capaldi (Ed.), Why we eat what we eat: The Psychology of eating (pp. 113-141). Washington DC: American Psychological Association.

Brehm, B. J., Seeley, S. R., Daniels, S. R., \& D’Alessio, D. A. (2003). A randomized trial comparing a very low carbohydrate diet and a calorie-restricted low fat diet on body weight and cardiovascular risk factors in healthy women. The Journal of Clinical Endocrinology \& Metabolism, 88(4), 1617-1623. doi:10.1210/jc.2002-021480

Capaldi, E. D. (1996). Conditioned food preferences. In E. D. Capaldi (Ed.), Why we eat what we eat: The Psychology of eating. (pp. 53-80). Washington, DC: American Psychological Association.

Church, T. S., Martin, C. K., Thompson, A. M., Earnest, C. P., Mikus, C. R., \& Blair, S. N. (2009). Changes in weight, waist circumference and compensatory responses with different Doses of exercise among sedentary, overweight postmenopausal women. PLoS ONE, 4(2), e4515. doi:10.1016/j.neulet.2014.04.016

Cowart, B. J. (1981). Development of taste perception in humans: Sensitivity and preference throughout the life span. Psychological Bulletin, 90(1), 43-73.

Donnelly, J. E., Honas, J. J. Smith B. K., Mayo, M. S., Gibson, C. A., Sullivan, D. K., Lee, J., Herrmann, S. D., Lambourne, K., \& Washburn, R. A. (2013). Aerobic Exercise Alone Results in Clinically Significant Weight Loss for Men and Women: Midwest Exercise Trial 2. Obesity, 21(3), E219-E228. doi:10.1002/oby.20145

Dwyer-Lindgren, L., Freedman, G., Engell, R. E., Fleming, T. D., Lim, S. S., Murray, C. J. L. \& Mokdad, A. H. (2013). Prevalence of physical activity and obesity in US counties, 2001-2011: a road map for action. Population Health Metrics, 11(1), 7.

Fine, E. J., Segal-Isaacson, C. J., Feinman, R. D., Herszkopf, S., Romano, M. C., Tomuta, N. ... Sparano, J. A. (2012). Targeting insulin inhibition as a metabolic therapy in advanced cancer: A pilot safety and feasibility dietary trial in 10 patients. Nutrition, $28(10)$, 1028-1035. doi:10.1016/j.nut.2012.05.001

Fothergill, E., Guo, G., Howard, L., Kerns, J. C., Kunth, N. D., Brychta, R., Chen, K. Y., Skarulis, M. C., Walter, M., \& Hall, K. D. (2016). Persistent metabolic adaptation 6 years after "The Biggest Loser" competition. Obesity, 24(8), 1612-1619. doi:10.1002/oby.21538

Fung, J. (2016). Obesity code: Unlocking the secrets of weight loss. Greystrone Books.

Galef, J. G. (1991). A contrarian view of the wisdom of the body as it relates to dietary self-selection. Psychological Review, 98(2), 218-223. doi:10.1037/0033-295X.98.2.218

Gibson, A. A., Seimon, R. V., Lee, M. Y., Ayre, J., Franklin, T. P., Markovic, T. P. ...Sainsbury, A. (2015). Do ketogenic diets really suppress appetite? A systematic review and meta-analysis. Obesity Reviews, 16, 64-76. doi:10.1111/obr.12230

Griffit, R., Lluberas, R., Luhrmann, M. (2013). Gluttony in England? Long-term change in diet. The Institute for Fiscal Studies. doi:10.1920/BN.IFS.2012.00142

Gutiérrez, J. P., Rivera-Dommarco, J., Shamah-Levy, T., Villalpando-Hernández, S., Franco, A., Cuevas-Nasu, L. ...Hernández-Ávila, M. (2013). Encuesta nacional de salud y nutrición: Resultados nacionales (2a. ed.). (I. N. (MX), Ed.) Cuernavaca, México.

Houk, J. C. (1988). Control strategies in physiological systems. FASEB Journal, 97-107.

Imes, C. C. \& Burke, L. E. (2014). The obesity epidemic: The USA as a cautionary tale for the rest of the world. Current Epidemiology Reports, 1, 82-88.

I-Min, L., Djoussé, L., Sesso, H., Lu, W., \& Buring, J. E. (2010). Physical activity and weight gain prevention. JAMA, 303(12), 1173-1179.

Kirkorian, R., Shidler, M. D., Dangelo, K., Couch, S. C., Benoit, S. C., \& Clegg, D. J. (2012). Dietary ketosis enhances memory in mild cognitive impairment. Neurobiology of Aging, 33, 425.e19-425.e27. doi:10.1016/j.neurobiolaging.2010.10.006

Leibel, R. L., Rosenbaum, M., \& Hirsch, J. (1995). Changes in energy expenditure resulting from altered body weight. The New England Journal of Medicine, 332(10), 621-628 
Lustig, R. H. (2012). Fat chance: The hidden truth about sugar, obesity and disease. Londres: Harper-Collins, Publishers.

Lustig, R. H. (2013). Sugar has 56 names. Nueva York: Penguin.

Lustig, R. H. (2017). The hacking of the american mind. Nueva York: Avery.

Lutas, A. \& Yellen, G. (2013). The ketogenic diet: Metabolic influences on brain excitability and epilepsy. Trends in Neurosciences, 36(1), 32-40. doi:10.1016/j.tins.2012.11.005

Marvopoulos, J. C., Yancy, W. S., Hepburn, J., \& Westman, E. C. (2005). The effects of a lowcarbohydrate, ketogenic diet on the polycystic ovary syndrome: A pilot study. Nutrition \& Metabolism, 2(1), 35. doi:10.1186/1743-7075-2-35

McFarland, D. J. (1971). Feedback mechanisms in animal behaviour. Nueva York: Academic Press.

McTiernan, A., Sorensen, B., L. Irwin, M., Morgan, A., Yasui, Y., Rudolph,R. E., ... Potter, J. D. (2007). Exercise effect on weight and body fat in men and women. Obesity, 15(7), 1496-1512. doi:10.1038/oby.2007.178

Paoli, A. (2014). Ketogenic Diet for Obesity: Friend or Foe? International Journal of Environmental Research and Public Health, 11, 2092-2017. doi:10.3390/ijerph110202092

Paoli, A., Rubini, A., Volek, J. S., \& Grimaldi, K. A. (2013). Beyond weight loss: A review of the therapeutic uses of very-low-carbohydrate (ketogenic) diets. European Journal of Clinical Nutrition, 67, 789796. doi:10.1038/ejen.2013.116

Ramsay, S. D., Seeley, R. J. Bolles, R. C. \& Woods, S. C. (1996). Ingestive homeostasis: The primacy of learning. In E. D. Capaldi (Ed.), Why we eat what we eat: The Psychology of eating. (pp. 11-27). Washington, DC: American Psychological Association.

Rowland, N. E., Li, B. H., \& Morien, A. (1996). Brain mechanisms and the physiology of feeding. In E. D. Capaldi (Ed.), Why we eat what we eat: The Psychology of eating (pp. 173-204). Washington, DC: American Psychological Association.

Rozin, P. (1996). Sociocultural influences on human food selection. In E. D. Capaldi (Ed.), Why we eat what we eat: The Psychology of eating (pp. 233-263). Washington DC: American Psychological Association.

Rubini, A., Bosco, G., Lodi, A., Cenci, L., Parmagnani, A., Grimaldi, ... Paoli, A. (2015). Effects of twenty days of the ketogenic diet on metabolic and respiratory parameters in healthy subjects. Lung, 193(6), 939-945. doi:10.1007/s00408-015-9806-7

Sacks, F. M., Bray, G. A., Carey, B. J., Smith, S. R., Ryan, D. H., Anton, S. D., ... Williamson, D. A. (2009). Comparison of weight-lossdiets with different compositions of fat, protein, and carbohydrates. The New England Journal of Medicine, 360(9), 859-873.

Sainsbury, E., Kizirian, N. V., Partridge, S. R., Gill, T., Colaguiri, S., \& Gibson, A. A. (2018). Effect of dietary carbohydrate restriction on glycemic control in adults with diabetes: A systematic review and meta-analysis. Diabetes Research and Clinical Practice, 139, 239 252. doi:10.1016/j.diabres.2018.02.026

Samaha, F. F., Iqbal, N., Seshardi, P., Chicano, K. L., Daily, D. A., McGrory, J., ...Stern, L. (2003). A LowCarbohydrate as Compared with a Low-Fat Diet in Severe Obesity. The New England Journal of Medicine, 348(21), 2074-2081.

Schafe, G. E. \& Bernstein, I. L. (1996). Taste aversion learning. In E. D. Capaldi (Ed.), Why we eat what we eat: The Psychology of eating. (pp. 31-51). Washington DC: American Psychological Association.

Shai, I., Schwarzfuchs, D., Henkin, Y., Shahar, D. R., Witkow, S., Greenberg,I., ... Stampfer,M. J. (2008). Weight loss with a low-crbohydrate, mediterranean, or low-fat diet. The New England Journal of Medicine, 359(3), 229-241. doi:10.1186/1478-7954-10-22

Sumithran, P., Prendergast, L. A., Delbridge, E., Purcell, K., Shulkes, A., Kriketos, A., \& Proietto, J. (2013). Ketosis and appetite-mediating nutrients and hormones after weight loss. European Journal of Clinical Nutrition, 67(7), 759-764. doi:10.1038/ejen.2013.90

Swinburn, B. A., Sacks, G., Hall, K. D., McPhearson, K., Finewood, D. T., Moodie, M. L., \& Gartmaker, S. L. (2011). The global obesity pandemic: Shaped by global drivers and local environments. Lancet, 378, 804-814. doi:10.1016/S0140-6736(11)60813-1

Taubes, G. (2007). Good calories, bad calories. Toronto: Alfred Knopf.

Taylor, M. K., Sullivan, D. K., Mahnken, J. D., Burns, \& Swerdlow, R. H. (2018). Feasibility and efficacy data from a ketogenic diet intervention in Alzheimer's 
disease. Alzheimer's \& Dementia: Translational Research \& Clinical Interventions, 4, 28-36. doi:10.1016/j.trci.2017.11.002

Volek, J. S., Phinney, S. D., Forsythe, C. E., Quan, E. E., Wood, R. J. Puglisi, M. J. ... Feinman, R. D. (2009). Carbohydrate restriction has more favorable impact on the metabolic syndrome than low fat diet. Lipids, 44, 297-309. doi:10.1007/s1745-008-3274-2

WHO. (2018). World health statistics 2018: Monitoring health for the SDGs, sustainable development goals. Ginebra: World Health Organization. Retrieved Febrero 7, 2019, from https://apps.who.int/iris/bitstream/handle/10665/2725 96/9789241565585-eng.pdf?ua=1

Wilson, J. \& Lowery, R. (2017). The ketogenic bible: The authoritative guide to ketosis. Canada: Victory Belt Publishing Inc.

Yancy Jr., W. S., Olsen, M. K., Guyton, J. R., Bakst, R. P., \& Westman, E. C. (2004). A low-carbohydrate, ketogenic diet versus a low-fat diet to treat obesity and hyperlipidemia. Annals of Internal Medicine, 140(10), 769-777. doi:10.7326/0003-4819-140-10-20040518000006 